\title{
Path Analysis On the Cultivation of Humanistic Spirit in Medicine
}

\author{
Zhao Wei ${ }^{1, a}$, Cao Hongwei ${ }^{2, b *}$ and Sun Fengxian ${ }^{3, c}$ \\ ${ }^{1}$ MuDanJiang Medical University in HeiLongJiang Province, China \\ ${ }^{2}$ MuDanJiang Medical University in HeiLongJiang Province, China \\ ${ }^{3}$ MuDanJiang Medical University in HeiLongJiang Province, China \\ a zw16598@163.con, ${ }^{\mathrm{b}}$ 82611159@qq.com, ${ }^{\mathrm{c}}$ 59915603@qq.com
}

Key words: people-oriented; humanistic spirit; medical science; humanistic quality

\begin{abstract}
People-oriented, is actually a concrete manifestation of modern human spirit, humanistic spirit in modern medicine can be embodied in the concept of "patient-oriented", the humanistic spirit of "people - oriented" in medicine is an important means to prevent the "alienation of medicine". Modern medical model calls for the transformation of education concept in modern medicine, it will be the training objective of each educator in medical science, through the organic integration of medical science and humanistic spirit, to cultivate medical talents not only of solid knowledge in medicine but also having a heart fulfilled with humanistic spirit.
\end{abstract}

\section{Introduction}

The concrete content of the respect for patients in the new medical model is to value the patient's life and to restore the patient's health with the rational use of modern medical technology; to maintain the patient's personality and dignity, and to treat patients fairly with equal and reasonable medical rights is the formal reflection of the respect for patients. Cultivating humanistic spirit and carrying out the concept of People - oriented will be the inner motivation and soul of the humanistic spirit in modern medicine.

The basic requirements for medical workers to reflect humanistic spirit in medicine are as following: to respect for the patient's life value, to respect for the patient's personality and dignity, to respect for the patient's medical rights, which is the embodiment of "people-oriented" modern medical model. Medicine can be a learning of "benevolence", of which "virtuous" should be as the core; medicine can also be called human science, of which the purpose should be "people-oriented". The humanistic spirit or humanitarian spirit in medicine is not only supposed to be eternal, but also the more the development of the medical science, the more it should be fully embodied [1].

\section{Current situation about the lack of humanistic spirit in medicine}

The purpose of medicine is to treat patients who are struggling in pain and suffering from physical and mental illness. To save lives and relieve the pain, which is not only to be solved by high-tech medical skills, but also there is demand of humanistic spirit of life care and respect for the patient's personality, there will be a delicate relationship between the doctor and patient when the doctor with medical ethics has to choose whether the money or the patient.

However, the current medical ethics has encountered a serious landslide caused by the gradually lost of humanistic spirit in modern medical science under the impact of developed technology and the value view of interests above everything. On one hand, "science and technology first" is of 
dehumanization. Advanced medical equipment and medicine indicates the modernization of medical technology, it is also an important condition by which human health can be improved. However, "medical alienation" caused by the perfectionism of technology has led to the result that doctors disobey the basic principles of "science and technology centering on the needs of people" in the course of treatment, pay too much attention to the disease and ignore the patient's normal feelings [2]. On the other hand, the lack of "medical ethics" tends to be caused by the idea of "money is omnipotent". The pursuit of economic interests has kept out the brightness of the human spirit and is put above the moral [3]. At present, the compensation mechanism of public hospitals in our country is not perfect yet, especially the hospital has to rely on "income" to make up for losses and seek for development in the case of serious shortage of government financial investment, so that the hospital has formed a compensation mode that seems neither scientific nor normal but comparatively "reasonable", which induced the abnormal development of medical behavior and led to a very prominent contradiction, that is for the broad masses of the people, it is "expensive and difficult to see a doctor "[4]. More and more medical malpractice and medical disputes were caused by misdiagnosis due to the profit-making behavior of the hospitals and doctors, drugs and medical equipment sales rebate, so that people has unknowingly tasted the bitter fruit of the gradual fade of humanistic spirit in medicine in the course of "medical alienation".

People are paying more and more attention to human social attributes in modern medicine, the demand of human is not only the health of the body, but more attention to the better respect for human nature and personality, who the doctor is directly facing is the patient suffering from the disturbance and torture of the disease, the doctor's attitude and program is directly related to the patient's life and health, so doctor's respect for the patient's personality, humanistic care and humane service for the patient should be put in the first place. Medical colleges and universities are the cradle of nurturing doctors. The humanistic quality education in medicine is concerned with the morality cultivation of medical students. Starting from meeting the needs of society, emphasizing the service function of medical education and promoting the comprehensive development of medical students, has reflected the connotation of the scientific development view - "people - oriented".

\section{Curriculum for humanistic spirit cultivation in medicine}

Medical students we are cultivating today will be the medical talents needed for the future development of human health. Medical students in the future clinical practice are required not only to be able to heal the patient's physical illness, but also to master the patient's psychological illness. And to establish a good doctor-patient relationship in the course of treatment of the disease through psychological counseling, emotional stability, teaching medical knowledge, introduction of the disease, accurate treatment and other measures, so that patients can trust doctors, and doctors can be for the sake of patients, this is the only way to enhance the patient's confidence in the treatment of disease, and ultimately achieve the purpose of treatment. Therefore, to start from the humanistic quality education, to strengthen the healthy personality education and guidance of medical students, to cultivate their noble professional ethics and to reshape the humanistic spirit in medicine, will be the expectation of nowadays society [5]. By emphasizing the education of teaching medical students to respect the life in learning and daily life, students will be able to correctly understand their duties at the stage of moral quality preparation. 


\section{Humanistic Curriculum Must Be All-encompassing}

The courses of humanities and social sciences in higher medical colleges are set up according to the contents and characteristics of different classes and different courses. The arrangement of the curriculum of humanities and social sciences should form a series, and in accordance with the principle of continuously deepening and expanding, it will form the orderly relationship and according to the psychosomatic development of medical students and the educational goals of different stages, to determine the teaching content of different stages, and to make the humanities and social sciences course all-round, ladder-style throughout the course study of medical students, to carry out the humanistic quality education to the end.

\section{Focus On The Innovation Of Education}

The professional characteristics of medical education require teachers to integrate the attitude of cooperation, humanistic quality, sound physique and mentality, and noble medical ethics into professional innovation education to cultivate the innovative quality of medical students. Adhere to the principle of student as the main body, to firmly establish the thought of "student-oriented", all-round knowing of students, understanding students, serving students, the basic point of teaching and learning work depends on the needs of students and the development direction of talents; Besides, innovation in education requires creative teachers. Creative teachers must have a high degree of cultural commitment and innovative education concepts, with the spirit of the times and huge professional humanistic knowledge, a sense of responsibility and efficiency in teaching, paying attention to the use of creative teaching methods to develop the intelligence of medical students, seeing students even the whole society as their own research object, serving the human as the main purpose of medical education, implementing the principle of people-oriented and the purpose of serving the patient in the whole process of learning and future practice.

\section{The Mechanism Reform of Humanistic Spirit in Medicine}

To Make Revolution in the Teaching Evaluation Mechanism, to Estimate the Teaching Behaviors of Teachers By the Integration of Science and Humanistic Education in the Teaching As the Most Important Points of Teaching Evaluation.

Medical students relatively need to learn much more professional knowledge than students of other majors have to, easier said than done, it is not easy to train a high school student with no knowledge of medical science to become a qualified clinician with just five years time. The quantity of medical knowledge needs to be studied must be huge, which is really a difficulty to medical schools. Medical colleges and universities often start the school the earliest and take a holiday the latest each semester, the concept of humanistic education of medical students needs to be changed, to add additional humanities curriculum is a more effective but not the only one method. Can or not all the teachers bear some of the responsibility of humanistic education in lectures? Only the humanities and social science course teachers have the right to the ideological and political education of students? How can teachers of other professional courses only teach students the scientific knowledge but not the truth in life?

Therefore, it is important for the specialized teachers in medical colleges to integrate the moral education into the teaching of professional knowledge echoing teachers of the humanities and social science, to combine the scientific education and humanistic education and to make the education go back to the fundamental goal of quality education. First of all, attaching importance to the education 
of medical ethics in the aspect of school teaching, and then to gradually make it a subtle and imperative teaching atmosphere. Social science teachers can teach students knowledge of humanities and social science based on medical practice and talk about the life and the dreams of medical students in the teaching; professional teachers of medical science can combine the scientific education and humanistic education in the teaching, to teach medical knowledge and medical practice combining the reflection of the value of life, so that students will not only learn the professional knowledge but also know why they learn that and make use of the science with what kind of heart. Only by the integration of the scientific knowledge and humanistic spirit, medical students can grow up with psychosomatic health and better fulfill the social responsibilities.

\section{To Strengthen the Integration of Science Education and Humanistic education}

Science education and humanistic education are two aspects of education. Science education emphasizes the direct social function of education, such as skills and modern scientific knowledge; humanistic education, to a certain extent, embodies the nature and fundamental purpose of education, such as the meaning of life, the self-awareness of life value and the cultivation of social responsibility. Science education is the means to achieve the fundamental purpose of education, through the learning and master of knowledge and skills, to better realize own life value and contribute to the development of society. Practice has proved that education does not attach importance to the fundamental purpose of education and neglects the means of achieving the purpose of education are imperfect education, which is unfavorable for the cultivation of talents. People, who have scientific knowledge but make a violation of social morality, are often of greater harm to society than those who do not learn knowledge. Medical students shoulder the responsibility to save human lives. Can you say that the transmission of human nature and warmth is not important yet in the process of saving lives? The humanistic education, which should be combined with scientific education, is pulled apart due to the current medical education system in China, and the cut is so thorough that it makes the courses of medical students so full, of which $85 \%$ is professional courses of medical science, and only the poor $15 \%$ is assigned to the social science teachers, this is a little bit of courses related to the ideological and political lessons, which also has become the vanguard for medical schools to respond to the country's banner of reducing student study load. Humanistic courses are in such an embarrassing situation in medical colleges and universities that how can students in medical colleges and universities attach importance to humanistic and social science courses?

\section{To Create A High Quality Humanistic Environment in Colleges and Universities.}

It is a critical period for the youth to establish life view and world outlook during university, medical students will grow rapidly both in mind and body in that period. Education is not only limited to the classroom, the heart of the youth are often more influenced by what they constantly see and hear in the university period. In order to enhance the humanistic quality of medical students, to promote the construction of campus culture, to create a human environment that is conducive to thinking, testing and creating, will be the best soil for the cultivation of humanistic sprit of medical students. Most of these spoiled one-children seem like small eagles that have left their parents for the first time, they are usually taken care of and they seldom care about others. Due to the habit of attribution of the human, it is very necessary for medical students to integrate into a collective atmosphere that all agree at this time, therefore, the impact of high-grade cultural atmosphere of the campus on the minds of students cannot be discounted. To create a high grade environment in the campus will 
greatly affect the taste of the people cultivated by the school, but also will be the necessary conditions to cultivate students with humanistic and mental health. The campus environment influences the students bit by bit with inspiration, such as respect for the teacher, to advocate students to take a polite attitude to teachers in and outside the campus; to promote the friendship between teachers and students, to advocate teachers to go down the podium; furthermore, the purpose and quality of the education, to a large extent, is dependent on s educational environment of the school, the greening of the campus, the programs of the campus broadcasting, and various activities hold by student union (giving seats to others on bus, the month of saving water, the speech about social ethics, the discussion contest), Therefore, we must adhere to the principle of people-oriented, full respect for knowledge, full respect for talent, and strive to create a cultural atmosphere of unity, harmony, pioneering and innovative, and proactive, so that the humanistic quality of medical students will be improved by leaps and bounds.

In short, the purpose of humanistic quality education in medicine is to enable the medical students to establish humanistic spirit in medicine such as values of "people-oriented", vocational views of "life-saving" and so on. With the highly development of the society, medical talents cultivated should be the perfect talents by the unity of personalization and socialization. The purpose of humanistic spirit cultivation in medicine is to make medical students establish a new medical concept, a comprehensive understanding of the relationship between medicine and society, cultivate good moral feelings, regulate the noble moral behavior, and develop the clinical skills of medical students relying on humanistic knowledge and methods. Modern medical model calls for the transformation of modern medical education concept, based on the organic integration of the medical science and humanistic spirit, to cultivate medical personnel and talents with solid knowledge in medical science, skilled in technique and imbued with humanistic spirit [6], is the goal of each medical educator, but also the guiding principles of the teaching and learning in medical colleges and universities.

\section{References}

[1] Zhang Jun, "On the role of medical ethics in the promotion of humanistic spirit in medicine[J]", Chinese Journal of Medical Ethics, 2002, (3): 29.

[2]Meng LingTao, etc. "On the humanistic spirit cultivation of medical students under the perspective of traditional Chinese medicine culture [J]”, Chinese Journal of Medical Ethics, 2013, (2): 29: 226-227.

[3] Zhao Wei, Xiang Yueli, Xu Min. "On the cultivation of medical ethics in Humanistic quality education [J]”, Medical Review, 2009, (8): 2559-2560.

[4] Rui Jing, "Analysis on the status of financial investment in Anhui public hospitals above the county level [J]”, Health Economics Research, 2007,10: 27-28.

[5] Gao Ling, Ji Xiaojun, "Views on strengthening and improving the humanistic quality education of medical students[J]”, Journal of Inner Mongolia Medical College .2006.28 (20: 161-162.

[6] Zhao Wei, Zhao Yue, Na Xiaowei, “The materialization of doctor-patient relationships and the concept of people-oriented[J]”, Hospital Management Forum, 2012, (7): 10-12. 